\title{
1 Title: How clonal are bacteria over time?
}

3 Author: B. Jesse Shapiro ${ }^{1}$

5 Affiliation

6 1. Département de sciences biologiques, Université de Montréal, Montréal, QC H3C 3J7, $7 \quad$ Canada

$8 *$ jesse.shapiro@umontreal.ca

\section{Abstract}

13 Bacteria and archaea reproduce clonally (vertical descent), but exchange genes by recombination

14 (horizontal transfer). Recombination allows adaptive mutations or genes to spread rapidly within

15 (or even between) species, and reduces the burden of deleterious mutations. Clonality - defined

16 here as the balance between vertical and horizontal inheritance - is therefore a key microbial trait,

17 determining how quickly a population can adapt and the size of its gene pool. Here, I discuss

18 whether clonality varies over time and if it can be considered a stable trait of a given population. I

19 show that, in some cases, clonality is clearly not static. For example, non-clonal (highly

20 recombining) populations can give rise to clonal expansions, often of pathogens. However, an

21 analysis of time-course metagenomic data from a lake suggests that a bacterial population's past

22 clonality (as measured by its genetic diversity) is a good predictor of its future clonality. Clonality

23 therefore appears to be relatively - but not completely - stable over evolutionary time.

\section{Introduction}

26 Here, I revisit the question posed in the title of a classic paper by John Maynard Smith and

27 colleagues [1]: How clonal are bacteria, and more specifically how does clonality vary among

28 different microbial populations and over time? First, what do we mean by clonality? Perfectly

29 clonal bacteria replicate by cell division (vertical descent) and evolve by random mutations that

30 occur during DNA replication. In this theoretical population, there is negligible horizontal

31 transfer of DNA by recombination across the resulting tree of vertical descent. Very few (if any)

32 natural bacterial populations fit this idealized, theoretical definition of clonality. Or, as discussed

33 below, they might only fit it for a short amount of time. However, knowing where a bacterial 
34 population of interest happens to fall along a spectrum of clonality can help us understand its

35 biology, and even make predictions about its evolution.

36 The opposite of clonality is panmixis - a situation in which the rate of horizontal transfer

37 is higher than the rate of vertical cell division, resulting in random association (linkage

38 equilibrium) among loci in the genome [1,2]. However, rates of horizontal transfer

39 (recombination) vary widely across the genome, such that a population can be mostly clonal,

40 except for a few loci in the genome [3]. These loci came to be termed genomic islands - a

41 metaphor I will build upon below. Some of the first islands identified were called pathogenicity

42 islands because they contained virulence factors [4]. However, non-pathogenic environmental

43 bacteria also contain islands, conferring adaptation to different ecological niches. For example,

44 genes in Prochlorococcus genomic islands confer adaptation to light and nutrient conditions

$45[5,6]$. But islands need not confer niche adaptation to their host genome; they can be neutral to

46 host fitness or even detrimental, selfish parasites. Here, I define genomic islands broadly as any

47 piece of DNA that is transferred horizontally (by either homologous or nonhomologous

48 recombination) from cell to cell and therefore evolves independently (i.e. is unlinked) from the

49 rest of the genome.

50 I will begin by extending the use of island analogies to include continents, peninsulas and

51 archipelagos (Table 1). I will then use these analogies to discuss to what extent microbial

52 populations are clonal or panmictic, and how often they transition between the two regimes.

\section{Are some islands really peninsulas?}

55 In the classic analogy, an island is totally disconnected from the mainland, meaning that genes in

56 the island evolve independently of the genome (Table 1). Examples of islands that fit this strict

57 independence might include integrated phages and other "selfish" elements, or genes that reside

58 in a particular niche but not in a particular genome (e.g. a gene ecology model [7]). Peninsulas

59 provide an analogy that might better describe how islands are related to microbial genomes. A

60 peninsula (or "presque-ile," from the French for "almost island") is a geographic term for a very

61 narrow strip of land connected to (but distinct from) the mainland. In my analogy, an island is

62 evolutionarily independent of the mainland genome, but their fates may become linked, forming a

63 peninsula. For example, a bacterium may acquire a gene from a vast microbial gene pool. This

64 gene allows the bacterium to invade a new ecological niche, triggering a clonal expansion in

65 which the fate of the gene and its new host genome are linked, at least for the duration of the

66 clonal expansion. One such example could be Yersinia pestis, which acquired a single gene

67 allowing flea-borne transmission and triggering a clonal expansion in the form of Plague 
68 pandemics [8]. Another peninsula, the prophage-encoded cholera toxin, and its links to the

69 mainland Vibrio cholerae genome [9,10], is discussed below.

Are some genomes archipelagos?

72 The very concept of one or a few islands implies a contrast with the large, clonal genomic

73 mainland or continent. But some microbial genomes may contain so many islands that there is no

74 mainland, only a vast archipelago (Table 1). A striking recent example is a population of

75 hotspring cyanobacteria in which virtually every gene in the genome evolved independently due

76 to frequent recombination [11], leading the authors to call the population "quasi-sexual" (in other

77 words, panmictic). Frequent recombination was confirmed by another group, using different

78 methods to study the same cyanobacteria [12]. This group also found that despite a history of

79 panmixis over long time scales, populations are more clonal over shorter time scales. Similarly,

80 the Asian ocean population of Vibrio parahaemolyticus also forms a panmictic gene pool, with

81 each recombination block of $\sim 1.8 \mathrm{kbp}$ evolving independently [13]. However, the panmictic gene

82 pool occasionally gives rise to pandemic clones. In another example, we found that almost every

83 gene in a population of Vibrio cyclitrophicus genomes showed signs of recombination over

84 relatively recent time scales [14]. Such apparently high rates of recombination in natural

85 populations were mysterious at first, contradicting recombination rates measured in the lab

$86[15,16]$ and predicted by theory $[7,17]$. However, theoretical models (discussed below) suggest

87 mechanisms capable of explaining how genes can spread through populations more rapidly by

88 recombination than by clonal expansion [18-20].

\section{Clonal expansions from panmictic pools}

91 I propose that archipelagos are not necessarily static over time, and that archipelagos can

92 sometimes coalesce into continents. Given the right ecological opportunity, a genome from a

93 panmictic gene pool can escape the "gravitational pull" of recombination and take off into a

94 clonal expansion. An example mentioned earlier is V. cholerae, a genetically diverse group of

95 coastal marine bacteria, some of which cause cholera. Virulence is mainly determined by two loci

96 in the genome: the cholera toxin and the toxin-coregulated pilus. Both genes are frequently gained

97 and lost by recombination [21,22], but are always found in one lineage of $V$. cholerae - the

98 lineage causing severe disease with pandemic potential, known as the phylocore genome (PG)

99 group [10]. It remains a mystery why the PG lineage evolved once, and only once. If PG $V$.

100 cholerae really did evolve just once, this would be surprising because $V$. cholerae draws on a

101 diverse, global gene pool and can be considered panmictic [23]. Therefore multiple different 
102 lineages would be expected to acquire the two (or perhaps a handful of) genetic elements required

103 for pandemic disease. This leads to the hypothesis that pandemic cholera emergence is selection

104 limited rather than diversity limited. In other words, benign $V$. cholerae strains constantly acquire

105 virulence genes. However, these strains rarely encounter the right ecological niche to flourish,

106 e.g. a human population consuming brackish water. "The right niche" has appeared a few times in

107 human history: for example in India in the 1800s, when the Classical lineage evolved, and again

108 in Indonesia in the 1950s, when the El Tor lineage evolved [24]. When the right conditions

109 appear, the PG lineage, along with its virulence factors, takes off in a clonal expansions which

110 continue to wreak havoc today (e.g. cholera pandemics from the 1800 s to today, all caused by the

111 PG clonal group). The virulence factors, previously islands in an archipelago, became a peninsula

112 connected to the PG mainland. The linkage between virulence factors and PG remains imperfect

113 because different variants of the cholera toxin continue to flow in and out of the PG continent

$114[10,21]$; hence the toxin remains a peninsula, not firmly part of the mainland.

$115 \quad V$. cholerae is a particularly well-characterized example of a panmictic gene pool giving

116 rise to a clonal expansion, but similar evolutionary dynamics are seen in other pathogens as well

117 (e.g. V. parahaemolyticus described above [13]). Enterotoxigenic Escherichia coli (ETEC) seems

118 to behave similarly, with deep branches of the phylogeny obscured by frequent recombination

119 and plasmid exchange, but more recent branches experiencing mostly clonal descent, with tight

120 linkage between virulence factors and the genomic mainland [25]. These observations are

121 consistent with an ancient, panmictic gene pool giving rise to clonal expansions, which can last

122 for decades or centuries.

\section{The balance between recombination and selection}

125 Let us consider the evolutionary forces that determine clonality: natural selection and

126 recombination. The effect of recombination on clonality is straightforward: more recombination

127 means less clonality. The effect of natural selection is more complex, but is defined here simply

128 as a force which favors clonal expansions of adaptive mutants within an ecological niche.

129 Selection, as defined here, therefore includes ecological effects. When driven by ecological

130 selection, clonal expansions are called selective sweeps, in which one clone outcompetes all

131 others, purging genetic diversity in the population.

132 Recombination and selection interact to determine the clonality of a population.

133 Recombination rates depend both on the ability of DNA to enter a cell and be incorporated into

134 the genome (the baseline rate) and the ability of that DNA to be retained by a balance of genetic

135 drift and natural selection (the realized rate). Some bacteria, such as Helicobacter pylori, have 
136 realized recombination rates that are much higher than point mutation rates, exchanging at least

$13710 \%$ of their genome within a single four-year human infection [26]. Others, such as

138 Staphylococcus aureus [27,28] and Mycobacterium tuberculosis are decidedly more clonal [29-

139 31]. Recombination rates (both realized and baseline) vary widely across the genome. Of 10

140 pathogenic bacterial species studied, all had identifiable recombination 'hot' regions, although

141 their length, genomic location and gene content varied [3]. Genes of different functions had

142 different realized recombination rates, implying a role for natural selection on gene function in

143 determining whether newly acquired genes are retained.

\section{Modeling the recombination-selection balance}

146 When rates of recombination are relatively low compared to rates of natural selection on adaptive

147 genes within niches, entire genomes will sweep to fixation before they can be shuffled by

148 recombination. Following previous modelling work, $s$ is defined here as the selective coefficient

149 of a niche-adaptive allele, and $r$ is the recombination rate, per locus per generation [7,32,33].The

$150 s \gg r$ regime is well described in the Stable Ecotype Model [17], which predicts that most of the

151 genome will follow a single, clonal phylogeny. Genome-wide sweeps thus increase clonality and

152 can be considered a hallmark of clonal populations (Table 1, Figure 1A). In the $r>s$ regime,

153 individual genes (rather than entire genomes) will sweep to fixation (i.e. reach $100 \%$ frequency)

154 in ecological niches to which they are adapted, without affecting genetic diversity elsewhere in

155 the genome (Figure 1B). The first serious theoretical attempt to reconcile the observations of

156 gene-specific sweeps with low recombination rates was the "Adapt Globally, Act Locally" model

$157[18,20]$, in which globally adaptive genes (adaptive in multiple different niches) trigger genome-

158 wide sweeps within a niche before being transferred to the next niche. When viewed across

159 niches, the result is a gene-specific sweep. A recent model suggested another mechanism by

160 which these gene sweeps can occur at moderate (not unrealistically high) rates of recombination

161 [20]. In this model, a microbial habitat is bombarded with genetically maladapted migrants,

162 allowing gene sweeps to occur, although the adaptive allele never reaches $100 \%$ frequency due to

163 the constant input of migrants. In another model, Takeuchi et al. [19] show that gene sweeps can

164 occur when $r$ is either very high or - counter-intuitively - when $r$ is very low, but only when

165 negative frequency-dependent selection (NFDS) reduces the rate of genome-wide selective

166 sweeps. NFDS might be commonly imposed on bacteria and archaea by viral (phage) predation,

167 providing a selective advantage to rare alleles of phage receptor genes [34].

168 One parameter not thoroughly explored in any of these models is the effective population

169 size $\left(\mathrm{N}_{\mathrm{e}}\right)$. Populations with small $\mathrm{N}_{\mathrm{e}}$ are dominated by drift, and natural selection is inefficient. 
170 They may experience genome-wide sweeps independently of $r$ and $s$. As discussed below, $\mathrm{N}_{\mathrm{e}}$ is

171 probably an important determinant of clonality.

172

\section{Genome-wide and gene-specific sweeps in nature}

174 To date, empirical evidence for gene-specific and genome-wide sweeps has come mostly from

175 cross-sectional studies of a single population of genomes at a single point in time, with

176 recombination and selection inferred backward in time [11,13,14,35]. Sequencing microbial

177 genomes or metagenomes sampled over time - already a typical practice in genomic

178 epidemiology (e.g. $[28,36])$ - promises to elucidate the rates of gene-specific and genome-wide

179 sweeps in nature (Figure 1).

180 In a pioneering study, Bendall et al. [37] sampled a lake over nine years and followed

181 single-nucleotide polymorphism (SNP) and gene frequencies in 30 bacterial populations by

182 metagenomic sequencing. They inferred that one of the populations (Chlorobium-111) had

183 undergone a near-complete genome-wide sweep over the nine-year study, with most SNP

184 diversity purged genome-wide (Figure 1A). By identifying regions of the genome with

185 unexpectedly low diversity compared to the genome-wide average, they inferred that gene-

186 specific sweeps had occurred in six of the populations, but these sweeps occurred before the

187 beginning of the nine-year study. During the nine-year study, they observed examples "where a

188 few adjacent SNPs trended toward fixation while genome-wide diversity was maintained"

189 (Figure 1B). They took this observation as consistent with gene-specific selective sweeps, but did

190 not attempt to determine whether the sweeps were due to selection or drift. Similarly, the inferred

191 genome-wide sweep could have been caused by selection or drift.

192 Is the frequency of genome-wide sweeps controlled mainly by the balance of

193 recombination and selection, or could drift (controlled by the effective population size, $\mathrm{N}_{\mathrm{e}}$ ) play

194 an important role as well? In their Figure 2 legend, Bendall et al. observe that "populations with

195 many SNPs were not necessarily sequenced deeper than those with few SNPs." This statement

196 was simply meant to assure the reader that SNP calling was not biased by sequence coverage, but

197 it also suggests that microbial populations tend to be far from a standard neutral model with

198 constant population size (i.e. the Wright-Fisher coalescent [38]). Taking SNP density as a rough

199 measure of $\mathrm{N}_{\mathrm{e}}$ and sequence coverage as a rough measure of the census population size, the data

200 show that census population size is a poor predictor of $\mathrm{N}_{\mathrm{e}}$, and thus that populations are likely

201 impacted by population bottlenecks and/or selection - although it is difficult to distinguish

202 between them. 
As a whole, the study showed that both genome-wide and gene-specific sweeps can occur

204 in different microbial populations from the same environment. Whether microbial populations

205 behaved differently due to differences in their ecology (i.e. regime of natural selection) or in their

206 baseline recombination rates remains a question for future study; Cohan recently suggested that

207 ecological differences could be more important [39]. Specifically, he suggests that ecological

208 "generalists" could have more opportunities for diversification, and thus be relatively immune to

209 genome-wide sweeps, compared to ecological specialists. I suggest that these generalists may

210 simply have larger $\mathrm{N}_{\mathrm{e}}$, such that diversity is rarely purged by drift (e.g. bottlenecks), and that

211 diversity is mainly governed by selection and recombination. It is possible that ecological

212 generalists tend to have large $\mathrm{N}_{\mathrm{e}}$, or that $\mathrm{N}_{\mathrm{e}}$ and ecology exert independent effects (e.g. ecological

213 generalists can have low $\mathrm{N}_{\mathrm{e}}$ and still resist genome-wide sweeps). The fact that one genome-wide

214 sweep was observed over a nine year period suggests that such events might be relatively rapid

215 but rare (only observed in one of 30 populations). Meanwhile, gene-sweeps might be more

216 common historically (affecting six of 30 populations), but could take longer to proceed to

217 completion.

\section{Is clonality a stable trait?}

220 As described in the $V$. cholerae example, some pathogenic bacterial populations can switch

221 between panmictic and clonal lifestyles [11,13,14,19,25,34,35]. Therefore clonality can vary over

222 time, but how much and how often? To quantify the stability of clonality over time, not just in

223 pathogens but in free-living environmental bacteria, I re-analyzed the lake time-course of Bendall

224 et al. [37]. Because estimates of selection and recombination rates were not readily available for

225 this dataset, I defined clonality based on the total genetic diversity (SNP density) in a population,

226 which scales with $\mathrm{N}_{\mathrm{e}}$, and includes the effects of both drift and of genome-wide selective sweeps.

227 Frequent genome-wide selective sweeps $(s>>r)$ and/or small $\mathrm{N}_{\mathrm{e}}$ result in clonality. I identified

22820 "old, diverse" populations as those with a high density of SNPs (>1500 SNPs/Mbp) at the

229 beginning of the time-course. These populations are likely "old" because they have gone a

230 relatively long time since the last genome-wide purge of genetic diversity and are relatively non-

231 clonal. They include the six populations inferred to have undergone gene-specific sweeps [37].

232 The remaining ten populations were defined as "young, low-diversity," having more recently

233 experienced a genome-wide purge of diversity. The "old, diverse" populations have a relatively

234 low ratio of nonsynonymous (N) to synonymous (S) SNPs, suggesting large $\mathrm{N}_{\mathrm{e}}$ and ample time

235 for purifying selection to remove (mostly deleterious) nonsynonymous mutations (Figure 2). In

236 contrast, the "young, low-diversity" populations are more likely to have high N:S ratios, 
237 suggesting smaller $\mathrm{N}_{\mathrm{e}}$ and less time for purifying selection to act. The cutoff of $1500 \mathrm{SNPs} / \mathrm{Mbp}$

238 (0.15\% SNP density) is somewhat arbitrary, but seems to correspond to an inflection point in

239 Figure 2, and also falls squarely within the 0-0.30\% SNP density, above which $E$. coli evolution

240 appears to transition from clonal to recombining [40].

241 With young (presumably clonal, or low $\mathrm{N}_{\mathrm{e}}$ ) and old (less clonal, higher $\mathrm{N}_{\mathrm{e}}$ ) populations

242 thus defined, I asked whether "old, diverse" populations tended to maintain their diversity

243 through the 9-year period of the study. In their paper, Bendall et al. defined two alternative

244 population types: 1) those that maintained stable SNP diversity over nine years, and 2) those that

245 experienced significant fluctuations in diversity due to clonal expansions - defined when one, but

246 not all timepoints are dominated by a single allele ( $\geq 95 \%$ frequency) at $>40 \%$ of SNP sites in the

247 genome [37]. By definition, the 19 populations of the first type did not experience genome-wide

248 sweeps during the study, while the 11 populations of the second type did experience genome-

249 wide purges of diversity, which were transient in ten cases and apparently permanent in one case

250 (Chlorobium-111). Strikingly, 17 out of 20 "old, diverse" populations maintained their diversity

251 over the nine-year study, compared to only two out of ten "young, low-diversity" populations

252 (Fisher test, Odds Ratio $=19.4, P<0.001$ ). This result suggests that populations with a history of

253 genome-wide sweeps tend to experience subsequent genome-wide sweeps, and those that have

254 maintained genetic diversity in the past tend to maintain their diversity into the future. In other

255 words, clonality can be considered a relatively stable microbial trait. However, we must take care

256 in taking a genome-wide sweep as evidence for clonality $(s>r)$. In small effective population

257 sizes, genome-wide sweeps can occur due to drift and bottlenecks, independently of $s$ and $r$.

258 Therefore, many of "young, low diversity" populations (Figure 2) may have lost diversity over

259 nine years due to low $\mathrm{N}_{\mathrm{e}}$, not due to low $r$.

260

\section{History repeats itself}

262 It appears that pathogens are more likely than free-living bacteria to undergo clonal expansions,

263 due in part to their ecology and transmission dynamics [41]. Free-living aquatic bacteria, on the

264 other hand, seem to be more likely to live in large, panmictic populations and behave like

265 archipelagos $[11,13,14,37]$. If clonality is indeed a stable trait, this implies that history will repeat

266 itself, and that the future behavior of microbial populations can be predicted with some

267 confidence from their past behavior. Diverse populations tend to stay diverse. Clonal populations

268 (that experience frequent genome-wide sweeps) tend to stay clonal. But history is not doomed to

269 repeat itself forever. As we have seen, clonal expansions, such as pandemic $V$. cholerae, may

270 originate when a panmictic gene pool (an archipelago) coalesces into a clonal continent, with 
271 virulence factors linked as peninsulas. Many such pathogenic clones have been documented, with

272 life spans of decades to thousands of years $[25,28,42,43]$. Other pathogens, such as Streptococcus

273 pneumoniae, may retain their panmictic population structure throughout an outbreak [44-46].

274 Why some pathogens are clonal and others are panmictic is an open question, but surely depends

275 on the balance between recombination and selection, on the effective population size, and on the

276 time scales considered.

278 Acknowledgments. I am grateful to Rex Malmstrom, Yan Boucher, and Salvador Almagro-

279 Moreno for their thoughtful comments which improved the manuscript. I was supported by a

280 Canada Research Chair. 
Table 1. Extended island metaphors of microbial genome evolution.

\begin{tabular}{|c|c|c|c|c|}
\hline $\begin{array}{l}\text { Geographic } \\
\text { metaphor }\end{array}$ & $\begin{array}{l}\text { Genetic unit to } \\
\text { which the } \\
\text { metaphor applies }\end{array}$ & $\begin{array}{l}\text { Type of selective } \\
\text { sweep experience } \\
\text { by the unit }\end{array}$ & $\begin{array}{l}\text { Dominant mode } \\
\text { of genetic } \\
\text { transmission }\end{array}$ & Example \\
\hline Island & Gene & Gene-specific & horizontal & $\begin{array}{l}\text { genes in the } V \text {. cholerae } \\
\text { integron }[22,23]\end{array}$ \\
\hline Peninsula & Gene & Genome-wide & vertical (clonal) & $\begin{array}{l}\text { the cholera toxin gene, } \\
\text { acquired horizontally, then } \\
\text { linked to a clonal } V \text {. } \\
\text { cholerae genome }[9,21]\end{array}$ \\
\hline Continent & Genome & Genome-wide & vertical (clonal) & $\begin{array}{l}\text { clonal expansions of } S \text {. } \\
\text { aureus }[28], \text { M. tuberculosis } \\
{[31,43]}\end{array}$ \\
\hline Archipelago & Genome & Gene-specific & horizontal & $\begin{array}{l}\text { hotspring cyanobacteria [11], } \\
\text { ocean vibrios }[13,14] \text {, } \\
\text { pneumococcus }[44,46]\end{array}$ \\
\hline
\end{tabular}

283

284

285

286

287

288

289

290

291

292

293

294

295

296

297

298

299

300

301

302

303

304

305

306

307

308

309

310

311

312

313

\section{Figures}

Figure 1. Temporal dynamics of genome-wide and gene-specific selective sweeps inferred from metagenomic data. Genetic diversity can be measured by mapping metagenomic reads to a reference genome, identifying SNPs, and calculating the allele frequencies at each SNP position in the genome over time. The lowest possible genetic diversity occurs when a single allele is present in $100 \%$ of metagenomic reads. Alternatively, diversity could be defined in terms of gene presence/absence, based on relative coverage of a gene in the reference genome by metagenomic reads. (a) In a hypothetical genome-wide selective sweep, all positions in the genome tend toward low diversity. (b) In a hypothetical gene-specific selective sweep, only one or a few positions in the genome tend toward low diversity, while the rest of the genome maintains high or intermediate diversity.

Figure 2. Past diversity predicts future diversity. Based on data from Table 2 of Bendall et al. [37], the nonsynonymous to synonymous (N:S) SNP ratio was plotted against the total SNP density (SNPs per megabasepair) for each of 30 bacterial populations. A pseudocount of 1 was added to both $\mathrm{N}$ and $\mathrm{S}$ counts. These 30 populations were divided into 20 "old, diverse" populations ( $>1500 \mathrm{SNPs} / \mathrm{Mbp})$ and 10 "young, low-diversity" populations ( $<1500 \mathrm{SNPs} / \mathrm{Mbp})$. Each point represents one of the 30 populations, colored in black if diversity was maintained over a 9-year metagenomic time-course, or in red if it was not. Seventeen out of 20 "old, diverse" populations maintained their diversity over the 9-year study, compared to only 2 out of 10 "young, low-diversity" populations (Fisher test, Odds Ratio $=19.4, P<0.001$ ). The same result is obtained drawing a cutoff based on N:S rather than SNPs/Mbp: populations with N:S $<0.5$ tend to maintain their diversity, whereas those with $\mathrm{N}: \mathrm{S}>0.5$ tend to be purged of diversity over the 9 years (Fisher test, Odds Ratio $=5.94, P=0.042$ ). Consistent with previous observations that N:S depends on the evolutionary time available for purifying selection to act $[47,48], \mathrm{N}: \mathrm{S}$ is negatively correlated with SNPs/Mbp, a proxy for evolutionary time or the time since the last genome-wide purge of genetic diversity in this dataset (Pearson's correlation of $\log _{10}$ transformed data, $r=-0.81, P=5.6 \mathrm{e}-8)$. 


\section{References}

1. Smith JM, Smith NH, O'Rourke M, Spratt BG: How clonal are bacteria? Proc Natl Acad 318 Sci USA. 1993, 90: 4384-4388.

319 2. Guttman DS, Dykhuizen DE: Clonal Divergence in Escherichia coli as a Result of

3. Yahara K, Didelot X, Jolley KA, Kobayashi I, Maiden MCJ, Sheppard SK, et al.: The landscape of realized homologous recombination in pathogenic bacteria. Mol Biol Evol. 2016, 33: 456-471. doi:10.1093/molbev/msv237

4. Hacker J, Blum-Oehler G, Mühldorfer I, Tschäpe H: Pathogenicity islands of virulent bacteria: structure, function and impact on microbial evolution. Mol Microbiol. 1997, 23: 1089-1097.

5. Coleman ML, Sullivan MB, Martiny AC, Steglich C, Barry K, Delong EF, et al.: Genomic islands and the ecology and evolution of Prochlorococcus. Science. 2006, 311: 1768-1770.

6. Coleman ML, Chisholm SW: Ecosystem-specific selection pressures revealed through comparative population genomics. Proc Natl Acad Sci USA. 2010, 107: 18634-18639. doi:10.1073/pnas.1009480107

7. Shapiro BJ, Polz MF: Ordering microbial diversity into ecologically and genetically cohesive units. Trends Microbiol. 2014, 22: 235-247. doi:10.1016/j.tim.2014.02.006

* A comprehensive discussion of the roles of selection and recombination in structuring microbial diversity.

8. Rasmussen S, Allentoft ME, Nielsen K, Orlando L, Sikora M, Sjögren K-G, et al.: Early Divergent Strains of Yersinia pestis in Eurasia 5,000 Years Ago. Cell. 2015, 163: 571582. doi:10.1016/j.cell.2015.10.009

9. Waldor MK, Mekalanos JJ: Lysogenic conversion by a filamentous phage encoding cholera toxin. Science. 1996, 272: 1910-1914.

10. Chun J, Grim CJ, Hasan NA, Lee JH, Choi SY, Haley BJ, et al.: Comparative genomics reveals mechanism for short-term and long-term clonal transitions in pandemic Vibrio cholerae. Proc Natl Acad Sci USA. 2009, 106: 15442-15447. doi:10.1073/pnas.0907787106

$350 *$ Deep sequencing of 90 cyanobacterial marker genes reveals extensive recombination, with each recombination in a quasisexual bacterial population occupying a broad niche. Science. 2015, 348: 1019-1023. doi:10.1126/science.aaa4456 genome composed of a random mix of alleles from the gene pool. 
12. Melendrez MC, Becraft ED, Wood JM, Olsen MT, Bryant DA, Heidelberg JF, et al.:

Recombination Does Not Hinder Formation or Detection of Ecological Species of Synechococcus Inhabiting a Hot Spring Cyanobacterial Mat. Front Microbiol. 2016, 6: 544. doi:10.1128/AEM.72.1.723-732.2006

$360 *$ In-depth population genomic evidence of a panmictic ocean gene pool containing non-clonal ecological populations.

14. Shapiro BJ, Friedman J, Cordero OX, Preheim SP, Timberlake SC, Szabo G, et al.:

13. Cui Y, Yang X, Didelot X, Guo C, Li D, Yan Y, et al.: Epidemic Clones, Oceanic Gene Pools, and Eco-LD in the Free Living Marine Pathogen Vibrio parahaemolyticus. Mol Biol Evol. 2015, 32: 1396-1410. doi:10.1093/molbev/msv009 Science. 2012, 336: 48-51. doi:10.1126/science.1218198

15. Vulić M, Dionisio F, Taddei F, Radman M: Molecular keys to speciation: DNA polymorphism and the control of genetic exchange in enterobacteria. Proc Natl Acad Sci USA. 1997, 94: 9763-9767.

16. Majewski J, Cohan FM: DNA sequence similarity requirements for interspecific recombination in Bacillus. Genetics. 1999, 153: 1525-1533.

17. Wiedenbeck J, Cohan FM: Origins of bacterial diversity through horizontal genetic transfer and adaptation to new ecological niches. FEMS Microbiology Reviews. 2011, 35: 957-976. doi:10.1111/j.1574-6976.2011.00292.x

18. Majewski J, Cohan FM: Adapt globally, act locally: the effect of selective sweeps on bacterial sequence diversity. Genetics. 1999, 152: 1459-1474.

19. Takeuchi N, Cordero OX, Koonin EV, Kaneko K: Gene-specific selective sweeps in bacteria and archaea caused by negative frequency-dependent selection. $B M C$ Biology. 2015, 13: 20. doi:10.1186/s12915-015-0131-7

$379 *$ Mathematical modeling shows how individual genes can sweep through populations with low recombination rates in the presence of negative frequency-dependent selection.

381

382

20. Niehus R, Mitri S, Fletcher AG, Foster KR: Migration and horizontal gene transfer divide microbial genomes into multiple niches. Nature Communications. 2015, 6: 8924. doi:10.1038/ncomms9924 several waves of global transmission in the seventh cholera pandemic. Nature. 2011, 477: 462-U111. doi:10.1038/nature10392 of Genetic Interactions between Vibrio metoecus and Vibrio cholerae, Two Close Relatives Co-Occurring in the Environment. Genome Biology and Evolution. 2015, 7: 2941-2954. doi:10.1093/gbe/evv193/-/DC1 

as within species, and that $24 \%$ of 'core' genes have crossed species boundaries. Mobile Gene Pools Rapidly Cross Species Boundaries To Create Endemicity within Global Vibrio cholerae Populations. mBio. 2011, 2: e00335-10. doi:10.1128/mBio.00335-10

24. Boucher Y, Orata FD, Alam M: The out-of-the-delta hypothesis: dense human populations in low-lying river deltas served as agents for the evolution of a deadly pathogen. Front Microbiol. 2015, 6: 1120. doi:10.3389/fmicb.2015.01120

25. Mentzer von A, Connor TR, Wieler LH, Semmler T, Iguchi A, Thomson NR, et al.: Identification of enterotoxigenic Escherichia coli (ETEC) clades with long-term global distribution. Nature Genetics. 2014, 46: 1321-1326. doi:10.1038/ng.3145

26. Cao Q, Didelot X, Wu Z, Li Z, He L, Li Y, et al.: Progressive genomic convergence of two Helicobacter pylori strains during mixed infection of a patient with chronic gastritis. Gut. 2015, 64: 554-561. doi:10.1136/gutjnl-2014-307345

27. Vos M, Didelot X: A comparison of homologous recombination rates in bacteria and archaea. The ISME journal. 2009, 3: 199-208. doi:10.1038/ismej.2008.93

28. Baines SL, Holt KE, Schultz MB, Seemann T, Howden BO, Jensen SO, et al.: Convergent adaptation in the dominant global hospital clone ST239 of methicillinresistant Staphylococcus aureus. mBio. 2015, 6: e00080. doi:10.1128/mBio.00080-15

417 30. Namouchi A, Didelot X, Schock U, Gicquel B, Rocha EPC: After the bottleneck:

29. Hershberg R, Lipatov M, Small PM, Sheffer H, Niemann S, Homolka S, et al.: High Functional Diversity in Mycobacterium tuberculosis Driven by Genetic Drift and Human Demography. PLOS Biol. 2008, 6: e311. doi:10.1371/journal.pbio.0060311.st006

424 32. Shapiro BJ, David LA, Friedman J, Alm EJ: Looking for Darwin's footprints in the

31. Casali N, Nikolayevskyy V, Balabanova Y, Harris SR, Ignatyeva O, Kontsevaya I, et al.: Evolution and transmission of drug-resistant tuberculosis in a Russian population. Nature. 2014, 46: 279-286. doi:10.1038/ng.2878

427 33. Friedman J, Alm EJ, Shapiro BJ: Sympatric Speciation: When Is It Possible in microbial world. Trends in Microbiology. 2009, 17: 196-204. doi:10.1016/j.tim.2009.02.002

429 34. Cordero OX, Polz MF: Explaining microbial genomic diversity in light of evolutionary 430 ecology. Nature Reviews Microbiology. 2014, 12: 263-273. doi:10.1038/nrmicro3218 
431

432

433

434

435

436

437

438

439

440

441

442

443

444 38. Kingman J: On the genealogy of large populations. J Appl Prob. 1982, 19: 27-43.

445

446

447

448

449

450

451

452

453

454

455

456

457

458

459

460

461

462

463

464

465

466

467

35. Cadillo-Quiroz H, Didelot X, Held NL, Herrera A, Darling A, Reno ML, et al.: Patterns of Gene Flow Define Species of Thermophilic Archaea. PLOS Biol. 2012, 10: e1001265. doi:10.1371/journal.pbio.1001265.t001

36. Croucher NJ, Didelot X: The application of genomics to tracing bacterial pathogen transmission. Current Opinion in Microbiology. 2015, 23: 62-67. doi:10.1016/j.mib.2014.11.004

37. Bendall ML, Stevens SL, Chan L-K, Malfatti S, Schwientek P, Tremblay J, et al.: Genome-wide selective sweeps and gene-specific sweeps in natural bacterial populations. The ISME journal. 2016, advance online publication, 8 January 2016; doi:10.1038/ismej.2015.241

** A pioneering application of time-course metagenomics to infer genome-wide and genespecific selective sweeps in natural lake bacterial populations.

39. Cohan FM: Bacterial Speciation: Genetic Sweeps in Bacterial Species. Curr Biol. 2016, 26: R112-R115. doi:10.1016/j.cub.2015.10.022

40. Dixit PD, Pang TY, Studier FW, Maslov S: Recombinant transfer in the basic genome of Escherichia coli. Proc Natl Acad Sci USA. 2015, 112: 9070-9075. doi:10.1073/pnas.1510839112

41. Achtman M, Wagner M: Microbial diversity and the genetic nature of microbial species. Nature Reviews Microbiology. 2008, 6: 431-440. doi:10.1038/nrmicro1872

42. Snitkin ES, Zelazny AM, Thomas PJ, Stock F, NISC Comparative Sequencing Program, Henderson DK, et al.: Tracking a Hospital Outbreak of Carbapenem-Resistant Klebsiella pneumoniae with Whole-Genome Sequencing. Science Translational Medicine. 2012, 4: 148ra116-148ra116. doi:10.1126/scitranslmed.3004129

43. Comas I, Coscolla M, Luo T, Borrell S, Holt KE, Kato-Maeda M, et al.: Out-of-Africa migration and Neolithic coexpansion of Mycobacterium tuberculosis with modern humans. Nature Genetics. 2013, 45: 1176-1182. doi:10.1038/ng.2744

44. Croucher NJ, Finkelstein JA, Pelton SI, Mitchell PK, Lee GM, Parkhill J, et al.: Population genomics of post-vaccine changes in pneumococcal epidemiology. Nature Genetics. 2013, 45: 656-663. doi:10.1038/ng.2625

45. Chewapreecha C, Harris SR, Croucher NJ, Turner C, Marttinen P, Cheng L, et al.: Dense genomic sampling identifies highways of pneumococcal recombination. Nature Genetics. 2014, 46: 305-309. doi:10.1038/ng.2895

46. Marttinen P, Croucher NJ, Gutmann MU, Corander J, Hanage WP: Recombination produces coherent bacterial species clusters in both core and accessory genomes. Microbial Genomics. 2015, 1. doi:10.1099/mgen.0.000038. 
$468 *$ The first simulation of bacterial evolution including mutation, allelic exchange (homologous

469 recombination) and gene gain/loss (non-homologous recombination).

470

471 47. Rocha EP, Smith JM, Hurst LD, Holden MT, Cooper JE, Smith NH, et al.: Comparisons 239: $226-235$.

475

48. Kryazhimskiy S, Plotkin JB: The Population Genetics of dN/dS. PLOS Genet. 2008, 4: e1000304.

476 


\section{Figure 1}

(a) Genome-wide sweep

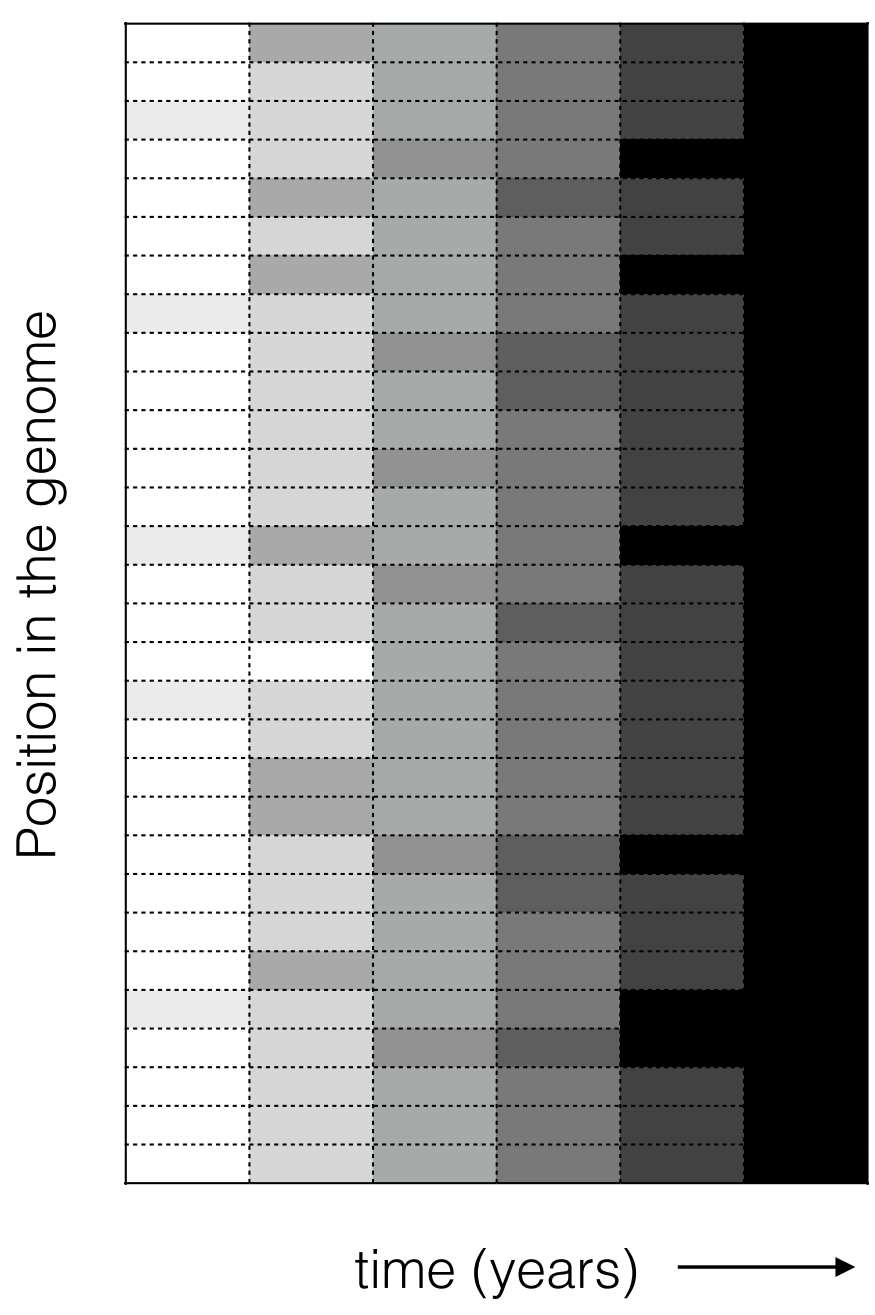

(b) Gene-specific sweeps

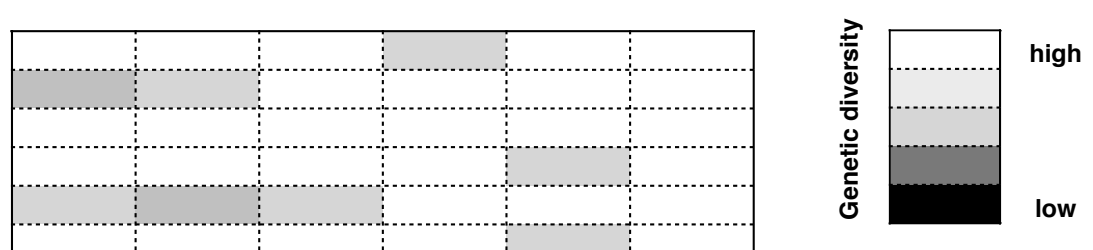

gene-specific sweep completed during the period of study $\leftarrow$ gene-specific sweep completed before the period of study 


\section{Figure 2}

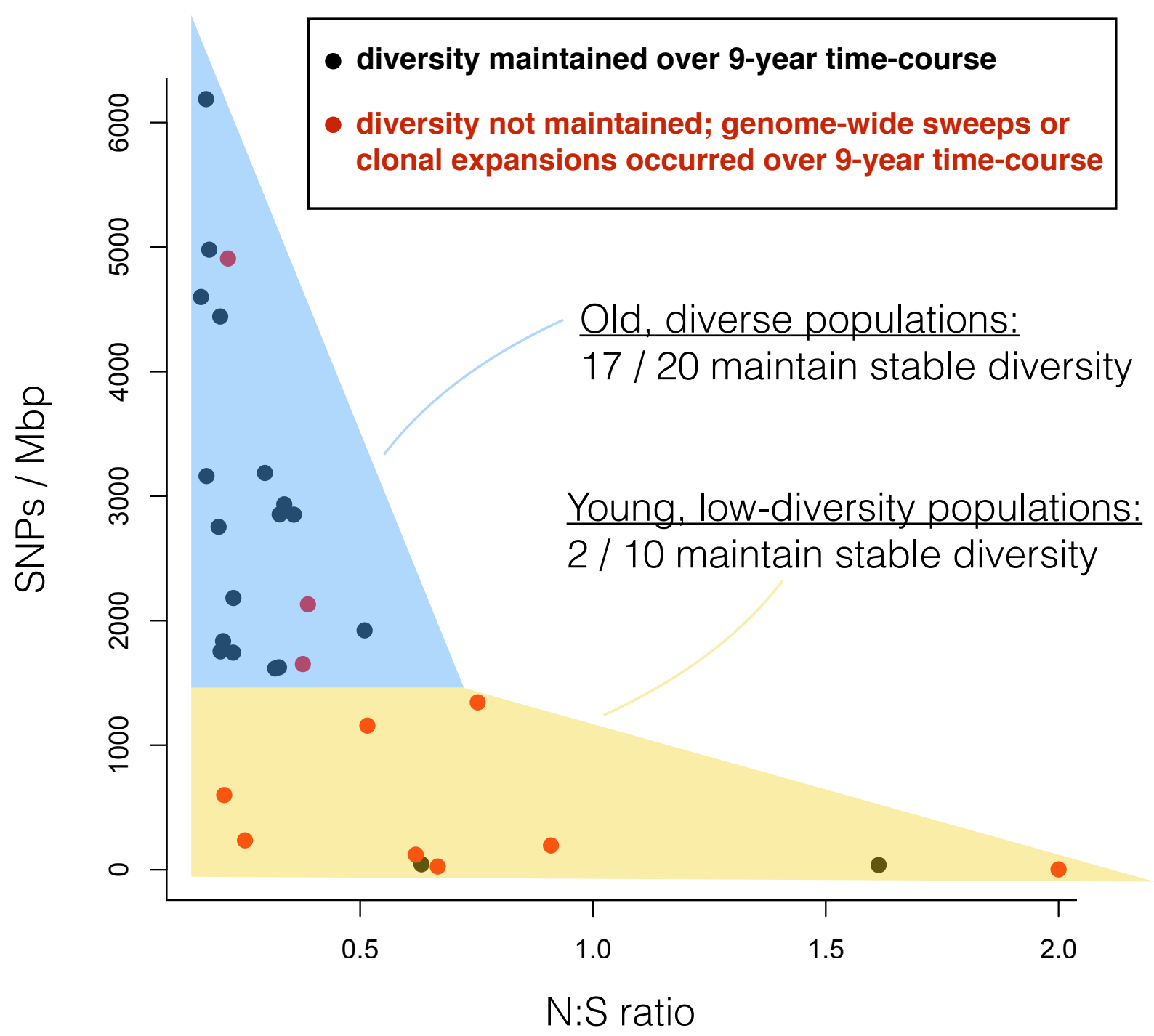

